\title{
Optical Mapping of Electrical Activity in Rat Somatosensory and Visual Cortex ${ }^{1}$
}

\author{
HARRY S. ORBACH, ${ }^{2}$ LAWRENCE B. COHEN, AND AMIRAM GRINVALD 3
}

Department of Physiology, Yale University School of Medicine, New Haven, Connecticut 06510, and Department of Neurobiology, Weizmann Institute of Science, Rehovot, Israel and The Marine Biological Laboratory, Woods Hole, Massachusetts 02543

\begin{abstract}
We have investigated the use of optical methods for monitoring neuron activity in mammalian cortex. The cortex was stained with a voltage-sensitive dye and fluorescence was simultaneously measured from 124 areas using a photodiode array. Optical signals were detected in rat somatosensory cortex in response to small whisker movements and in visual cortex in response to light flashes to the eye. Relatively large signals were obtained curing focal interictal epileptiform discharges induced by bicuculline. The measuring system had a time resolution of milliseconds and a spatial resolution of a few hundred micrometers. Simultaneous, multi-site optical recordings of activity may provide a new and potentially powerful method for studying function and dysfunction in mammalian cortex.
\end{abstract}

Understanding of cortical function would be enhanced by a more detailed monitoring of its activity. A useful method would (1) have a time resolution in the millisecond range; (2) have a spatial resolution $(\sim 100 \mu \mathrm{m})$ capable of resolving functional units, e.g., cortical columns, or gross features of sensory and motor maps; and (3) be able to monitor activity at many sites simultaneously. These goals might be achieved by using voltage-sensitive dyes which respond to changes in membrane potential by changing their absorption and/or fluorescence (Cohen and Salzberg, 1978; Waggoner, 1979).

Recent experiments have shown that optical measurements using a multi-element diode array can provide simultaneous mapping of activity from multiple regions of rat hippocampal slices (Grinvald et al., 1982a), the in vitro frog neurohypophysis (Salzberg et al., 1983), and in vitro and in vivo salamander olfactory bulb (Orbach and Cohen, 1983); for reviews see Cohen et al., 1978; Salzberg, 1983; Grinvald, 1985). Although these results suggested that optical methods might be used in the mammalian cortex, two questions had to be resolved. First, the earlier experiments did not use physiological

Received October 16, 1984. Revised December 27, 1984;

Accepted December 28, 1984

' We thank Charles Gilbert, Peter Hand, Ehud Kaplan, Torsten Wiesel, and Tom Woolsey for much helpful advice. We are grateful to Rina Hildesheim for the synthesis of the styryl dyes, and we thank Dr. A. S. Waggoner, Fluorescence Center, Carnegie-Mellon University, Pittsburgh, Pennsylvania, for providing the oxonol dyes. This work was supported by National Institutes of Health Grants NS 08437 and NS 14716, a United States-Israel Binational Science Foundation Grant, and a National Institutes of Health traineeship to H. S. O.

${ }^{2}$ Present address: Division of Biology, California Institute of Technology, Pasadena, CA 91125.

${ }^{3}$ Present address: Rockefeller University, New York, NY 10021. stimuli but relied on electrical stimulation to initiate activity. Physiological stimuli might result in a much smaller fraction of activated neurons and, thus, much smaller signals. Second, in vivo measurements on mammalian cortex could be more difficult because of artifacts resulting from the gross movements of the brain due to the heartbeat and respiration.

In the present experiments we investigated the cortical responses to whisker movements or light flashes to the eye in the rat. The upper layers of sensory cortex were stained with styryl or oxonol dyes (Gupta et al., 1981; Grinvald et al., 1982b) by placing a pool of dye solution on top of the cortex. A microscope objective was used to project an image of a region of cortex onto a 100- or 124element photodiode array. At the magnifications used, each detector received light from a $168 \times 168 \mu \mathrm{m}$ or a $264 \times 264 \mu \mathrm{m}$ area of the object plane. Thus, each detector received light from many neurons and processes; signals probably represent the summed responses from simultaneously active neurons (or glia). Because the movement artifacts resulting from the heartbeat were about the same from one heartbeat to the next, subtraction of recordings without stimuli from recordings with stimuli reduced the artifact.

Preliminary reports of these experiments have appeared (Orbach et al., 1982, 1983). Reports of more recent optical experiments of visually evoked responses in frog optic tectum (Grinvald et al., 1984) and preliminary results describing odor-induced signals in salamander olfactory bulb (Kauer et al., 1984) have been published.

\section{Materials and Methods}

A schematic drawing of the light path is illustrated in Figure 1A; the apparatus is identical to that described previously (Fig. $1 D$ of Orbach and Cohen, 1983; Grinvald et al., 1981a). Epi-illumination was used and fluorescent signals were measured since absorption-dependent, light-scattering signals (Ross et al., 1977) were small in experiments on salamander olfactory bulb (Orbach and Cohen, 1983). Light from a tungsten-halogen lamp was passed through a heat filter (not shown) and a wide-band interference filter (Fig. 1A, filter 1) before being reflected down onto the cortex by a dichroic mirror. For the styryl dyes we used an interference filter of $520 \pm 45 \mathrm{~nm}$ and a dichroic mirror with a half-reflectance wavelength of $570 \mathrm{~nm}$ (Omega Optical, Brattleboro, VT) or a Zeiss FT580. A Schott RG610 long-wavelength pass filter (Fig. 1A, filter 2) was used to block the scattered and reflected incident wavelengths while passing the longer-wavelength fluorescent light. The filters used for oxonol dyes were: filter $1(630 \pm 15 \mathrm{~nm}$ ), a dichroic mirror with $50 \%$ transmission at $650 \mathrm{~nm}$ or a Zeiss HPL half-silvered mirror, and filter 2, Schott RG 665. The smaller Stokes shift of oxonol dyes means that compromises have to be made in the choice of filters and mirrors. (The oxonol dyes used here do, however, provide signals at wavelengths suitable for excitation with a helium-neon laser.) Either a Leitz Ortholux II or a Zeiss Universal microscope was used. The microscope objective (Nikon $4 \times, 0.16$ numerical aperture (na), or Wild 10x, $0.4 \mathrm{na}$ ) was used to form an enlarged image of a portion of the cortex at the photodiode array. The actual magnifications were $\times 5.3$ and $\times 8.3$. The objectives were chosen for large numerical aperatures since, in epi-fluorescence, the amount of light reaching the photodiodes will, for a fixed magnification, be proportional to the fourth power of numerical aperture (Grinvald et al., 1982b). Either a 100-element or 


\section{A. APPARATUS}

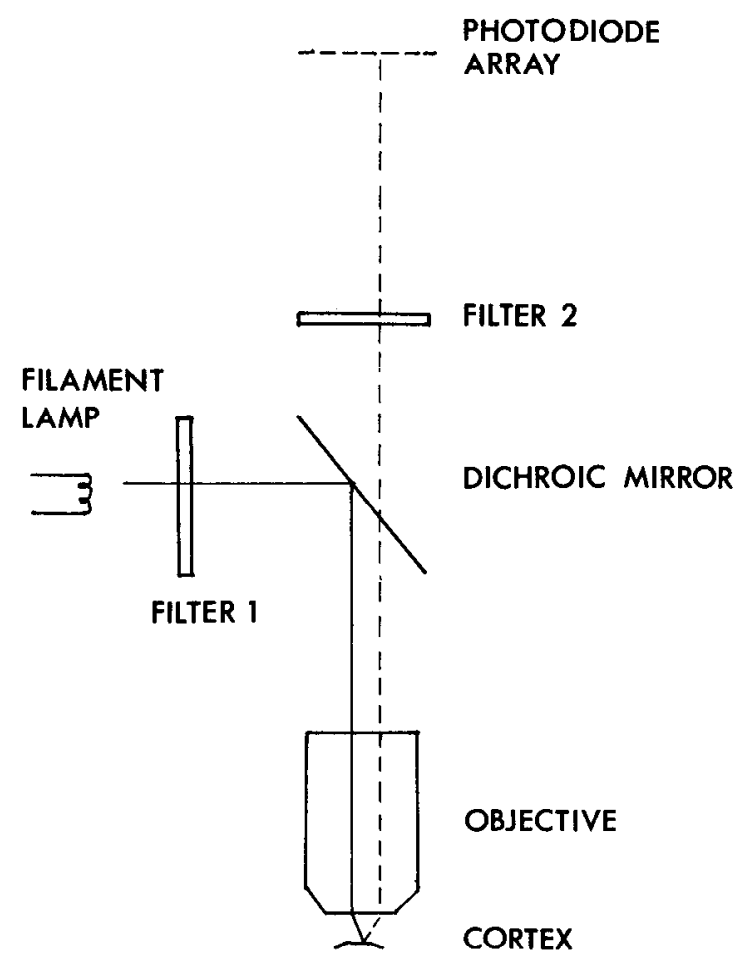

B. SINGLE TRIAL

\section{no subtraction}

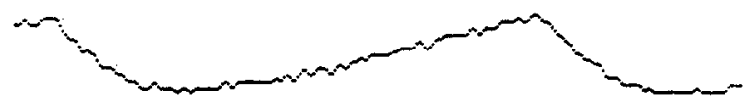

after subtraction

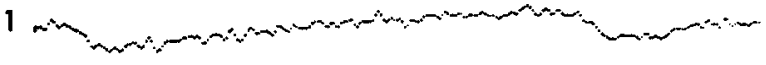

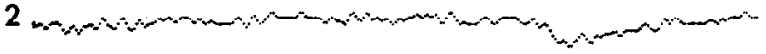

3

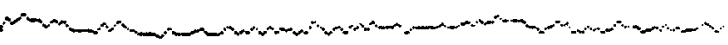

\section{FILTERING}

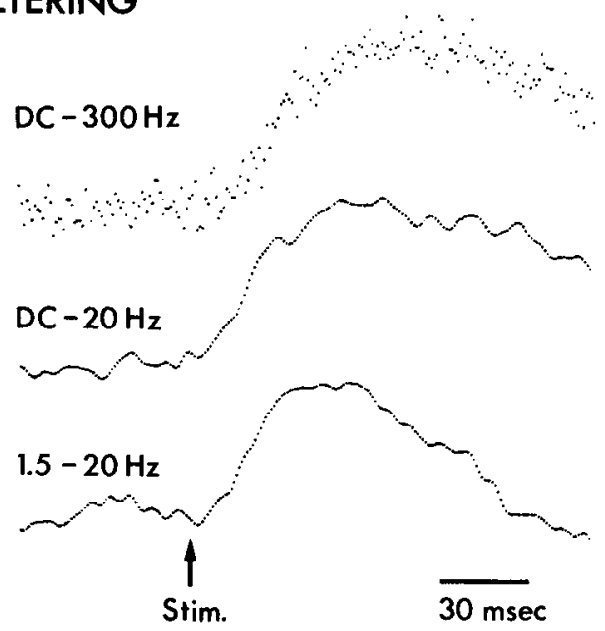

Figure 1. A, Schematic drawing of optical apparatus for epi-fluorescence. The photodiode array was placed at the plane where the objective makes a real, magnified, and inverted image. In many experiments the illumination intensity was increased by a factor of 2 by over-running the $12-V, 100-W$ tungstenfilament lamp (9 A instead of the nominal 8.3 A). B. Subtraction reduces noise locked to the electrocardiogram (but increases random and other non-locked noise). Four traces are illustrated: the top trace is a single recording without subtraction, and the bottom three traces are individual trials in which a recording was subtracted from its predecessor. $C$. Filtering to reduce noise. The top two traces are the same data but at different bandwidths. The third trace was from the subsequent measurement on the same preparation. The middle trace was obtained from the top trace by using 10 passes of a 1-2-1 digital filter. For each point in the trace, this filter took twice the value of the center point, added the result to the sum of the two side points, divided the total by four, and replaced the old center point with the result of the division. A program using a 21-point Fourier filter with Lanczos smoothing (Hamming, 1977), kindly provided by W. N. Ross and N. Lasser-Ross, gave a similar result. Comparison of the $D C-20 \mathrm{~Hz}$ trace and the $1.5-20 \mathrm{~Hz}$ trace docs not demonstrate a reduction in noise as a result of low frequency filtering. However, the AC coupling, by effectively subtracting out the large resting light intensity, allowed a larger gain in a second-stage amplifier, and this reduced the required digitization accuracy to 8 bits. In the way our systems were configured, 12 bit accuracy could only be obtained if half the number of detectors were used. In all experiments a heating pad was used to regulate the rectal ternperature between $35^{\circ} \mathrm{C}$ and $38.5^{\circ} \mathrm{C}$. No consistent differences in signal size were obtained over this temperature range. Staining was carried out at rectal temperatures between $31^{\circ} \mathrm{C}$ and $37^{\circ} \mathrm{C}$.

124 elements of a 144-element silicon photodiode array (Centronic Ltd., Craydon, United Kingdom) was used. Each element was $1.4 \times 1.4 \mathrm{~mm}$; the elements were separated by $0.1 \mathrm{~mm}$. With the $4 \times(\times 5.3)$ lens, each photodiode will receive light from a $264 \times 264 \mu \mathrm{m}$ area of the focal plane. With the $10 \times(\times 8.3)$ lens, this area will be $168 \times 168 \mu \mathrm{m}$. The output of each detector was amplified, digitized, and stored in a Digital Equipment Corp. PDP $11 / 34$ computer. The data were plotted at 10,000 points/sec on a Tektronix 4010 or $\mathbf{4 0 1 2}$ terminal using digital-to-analogue converters and the analogue-multiplexer modification of the terminal. These methods have been described in more detail (Grinvald et al., 1981a; Orbach and Cohen, 1983). The figures were made directly from the hard-copy output. Listings of the programs, assembly language subroutines called by Fortran, running under RT-11, are available from the authors.

Noise reduction. Three types of signal processing were used to reduce noise. The first type was designed to reduce the artifacts from the movement of the brain due to the heartbeat. Each recording period was triggered from the largest peak in the electrocardiogram to synchronize the beginning of the recording with the heartbeat. The top trace in Figure $1 B$ shows the output of one detector, in a recording triggered by a heartbeat, in which the next heartbeat occurred about $60 \%$ of the way through the sweep. The artifacts due to movement are large; the fractional changes $(\Delta F / F)$ were about $5 \times$ $10^{-3}$. However, if the artifacts are consistent from heartbeat to heartbeat, then subtraction of one recording from its predecessor should reduce this noise. Three examples of subtractions are shown in Figure $1 B$. In the first, the subtraction was imperfect for both heartbeats. In the second, the subtraction was good for the first heartbeat but not the second. In the third, the subtraction was good for both heartbeats. In all three, subtraction substantially reduced the heartbeat noise. In our experiments the stimulus was present in the first recording period but absent from the second recording period, and the diode outputs during the second period were subtracted from the outputs of the first period. This result is called one trial.

The noise that remains after heartbeat subtraction is larger than the shot noise (from random arrival of photons at the photodetector). There are probably three additional sources of noise. First, at the light intensities reaching the photodiodes, the dark noise was approximately equal to the shot noise. Second, the 8-bit resolution of the analogue-to-digital conversion may not have been adequate at the amplifier gains that were available, and, thus, inaccurate digitization may contribute to the noise. Third, there is presumably noise from movements that are not time locked to the heartbeat. Because of jitter in the time of the second heartbeat in the two recordings, there was often a movement artifact associated with the second heartbeat. All of the records, except Figure $1 B$, have been terminated just before the time that this artifact was expected.

Signal-averaging was usually used. The signal-averaging program included 
Figure 2. Optical detection of a localized region of activity. Simultaneous intensity changes were measured with 124 photodiodes from rat somatosensory cortex that resulted from a $1-\mathrm{mm}$ whisker movement. The signals are restricted to the central detectors of the array. The fractional changes in fluorescence, $(\Delta F / F)$, on the central four detectors are at least 20 times larger than the mean signal on all of the detectors around the very edge of the array. The time of the beginning of the whisker movement is indicated by the arrowheads below the traces in row 6 . In this figure and in Figure 4, top is medial, bottom is lateral, left is posterior, and right is anterior. The light intensity was largest in the center of the field and declined toward the edge. In this experiment some of the edge detectors received only half as much light as central detectors. Before staining, the amount of light reaching the photodiodes was lower by a factor of at least 10 . Thus, most of the light in the measurement results from dyerelated cortical fluorescence. The records were smoothed with 10 passes of the 1-2-1 digital filtering routine. The inset at the lower left shows the timing of the current to the galvanometer-stimulator. The missing traces in Figure 6 are the result of malfunctioning amplifiers. The whisker was moved in the caudal-to-rostral direction. Thirty-two trials were averaged. The interval between stimuli was $9 \mathrm{sec}$. The cortex has been stained with a $2-\mathrm{mg} / \mathrm{ml}$ solution of $\mathrm{RH} 414$ for 100 $\min$.
WHISKER, $1 \mathrm{~mm}$

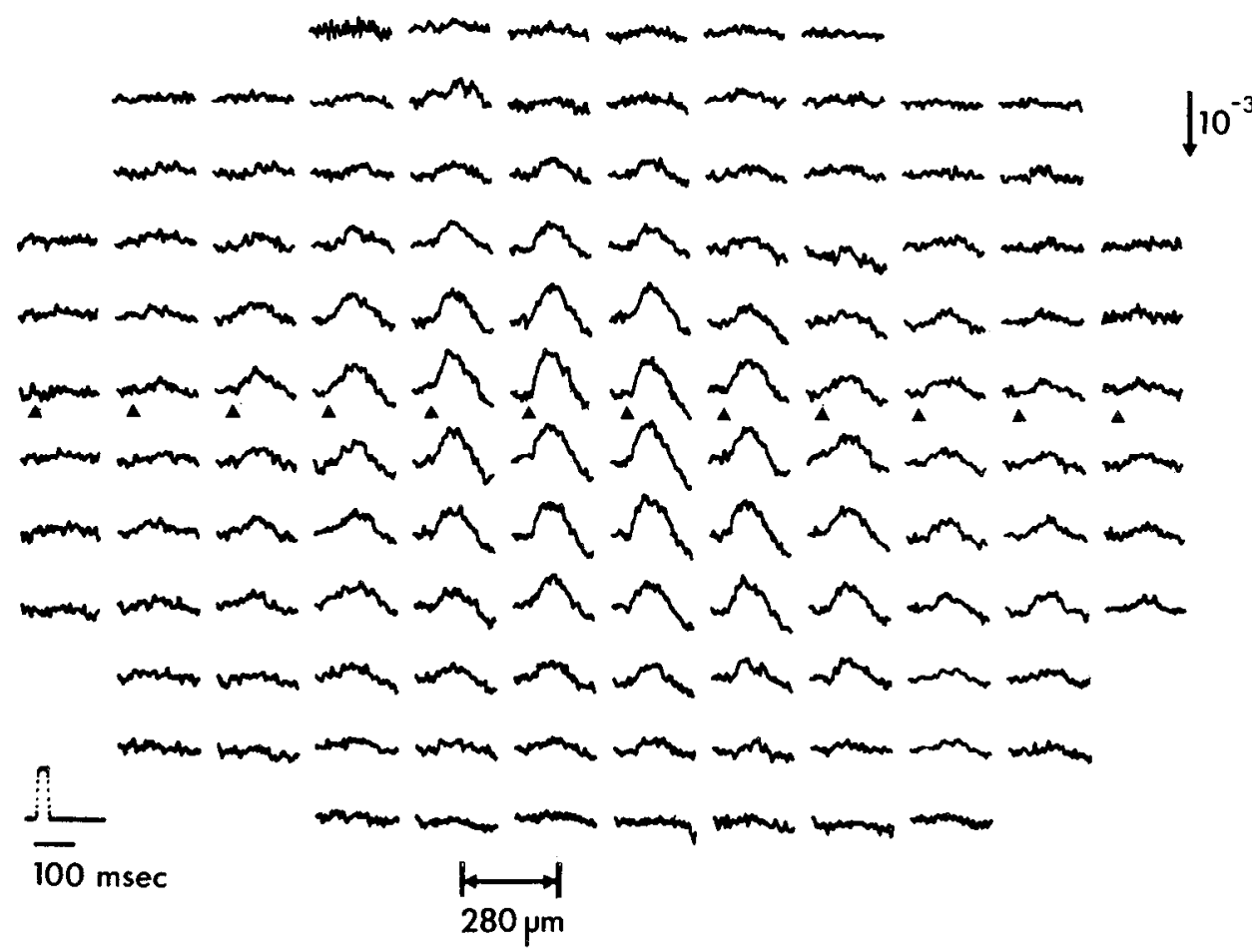

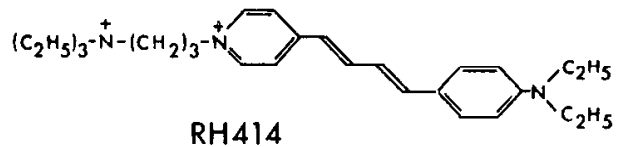

$\mathrm{RH} 414$

\section{${ }_{2} \mathrm{H}_{5}$}

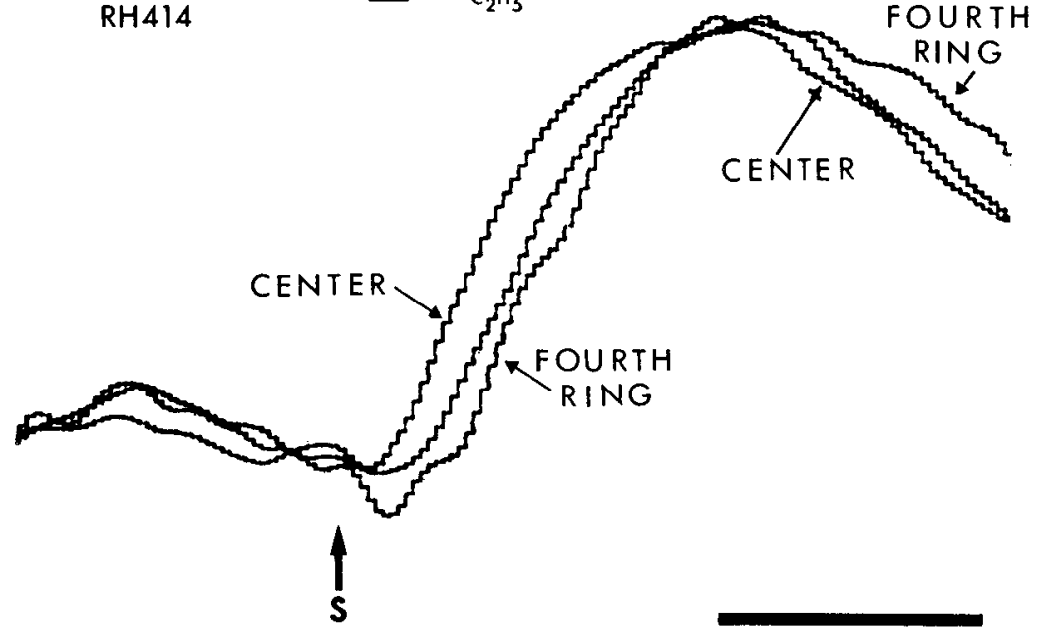

FOURTH

$30 \mathrm{msec}$

\begin{tabular}{|c|c|c|c|c|c|c|c|c|c|}
\hline & & & & 4 & & & & & \\
\hline & & 4 & 3 & 3 & 3 & & 4 & & \\
\hline & 4 & 3 & 2 & 2 & 2 & 2 & 3 & 4 & \\
\hline 4 & 3 & 2 & 1 & 1 & 1 & 1 & 2 & 3 & 14 \\
\hline 4 & 3 & 2 & 1 & $C$ & C & 1 & $z$ & & \\
\hline 4 & 3 & 2 & 1 & $C$ & C & 1 & 2 & 3 & 4 \\
\hline 4 & 3 & 2 & 1 & 1 & 1 & 1 & 2 & 3 & \\
\hline & 4 & 3 & 2 & 2 & 2 & 2 & 3 & 4 & \\
\hline & & 4 & 3 & 3 & 3 & 3 & 4 & & \\
\hline & & & & & & & & & \\
\hline
\end{tabular}

Figure 3. Time course of the signals from three different parts of the response area: the center, the edge (fourth ring), and an intermediate area (second ring). The signal had a shorter delay at the center and a longer delay at the edge. The inset, on the right, illustrates the arrangement of the central detectors (C) and the four rings of detectors (1 to 4) which we used. The signals illustrated are the sum of the four central detectors (C), the 16 detectors in ring 2 , and the 24 detectors in ring 4. The three signals were normalized to the same height. Since the response areas (e.g., Fig. 2) were often somewhat ellipsoidal rather than circular in shape, this kind of analysis can yield only approximate results. Sixty-four trials were averaged for the original data. The center is the sum of 4 detectors, the second ring is the sum of 16 detectors, and the fourth ring is the sum of 24 detectors. Whisker B1 was moved by 6 mm. Similar delays between center and edge were seen in other trials in which the whisker movement was only $1 \mathrm{~mm}$. The time of the beginning of the whisker movement is indicated by the arrow. The interval between trials was $5 \mathrm{sec}$. The cortex has been stained with a $4-\mathrm{mg} / \mathrm{ml}$ solution of RH 414 for $60 \mathrm{~min}$. The structure of $\mathrm{RH} 414$ (Grinvald et al., 1984) is shown above the traces. 


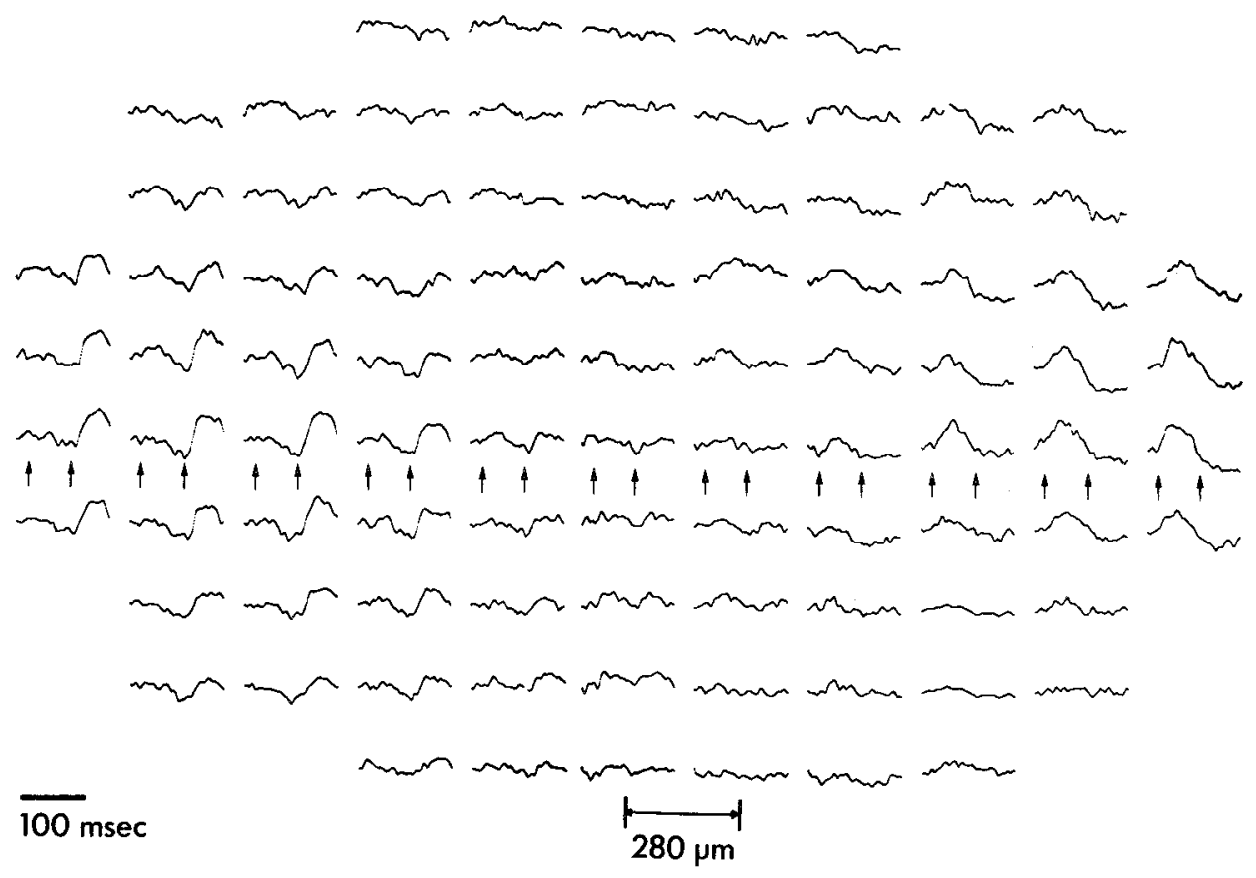

Figure 4. When two whiskers, relatively separated on the face, were stimulated, the response areas measured from somatosensory cortex were separated. Whisker D4 was moved by $3 \mathrm{~mm}$ early in the sweep; whisker A1 was moved by $2 \mathrm{~mm}$ later in the sweep. The time of the beginning of both movements is indicated by the arrows below the traces in row 6 . The records were smoothed by 20 passes of the 1-2-1 digital filter. The whiskers were moved in the caudal-to-rostral direction. A total of 128 trials were averaged. The interval between trials was $9 \mathrm{sec}$. The cortex had been stained with a $4-\mathrm{mg} / \mathrm{ml}$ solution of $\mathrm{RH}$ 414 for $50 \mathrm{~min}$. the possibility of rejecting trials with excessive noise. Trials in which the results of subtraction were not as good as trace 2 of Figure $1 B$ were usually rejected in addition to those trials which had very large movement artifacts due to coughs or sneezes. In most experiments 10 to $30 \%$ of the trials were rejected.

The low and high frequency bandwidths were limited electronically, before digitization. Additional high frequency filtering was carried out digitally. The low frequency bandwidth restrictions resulted in distortion of the signal time course. The records in Figure $1 C$ show the results of an experiment in which 64 trials were averaged in a measurement from somatosensory cortex. A whisker was moved by about $5 \mathrm{~mm}$, starting at the time of the arrow (Fig. $1 C)$. The top trace shows a measurement made with a bandwidth of DC$300 \mathrm{~Hz}$. The high frequency filtering of $300 \mathrm{~Hz}$ was provided by two parallel R-C circuits (time constants of $400 \mu \mathrm{sec}$ ) in the photodiode amplifiers. These filters were present in all experiments. The second trace in Figure $1 \mathrm{C}$ shows the same data but after 10 passes of a 1-2-1 digital smoothing routine which reduced the high frequency cutoff to approximately $20 \mathrm{~Hz}$. The third trace shows the result of another experimental run on the same preparation, in which low frequency filtering (capacity coupling with a time constant of 100 msec in the diode amplifiers) was also used. Clearly, this filtering distorts the falling phase of the signals. Except for the top two traces in Figure $1 \mathrm{C}$, all of the recordings presented in this paper have used this low frequency filtering

Because it was our impression that focusing the objective at the surface of the cortex increased the noise, all of the experiments were done with the objective focused at a level 200 to $300 \mu \mathrm{m}$ below the cortical surface.

Preparation. Albino (somatosensory cortex) or Long-Evans (visual cortex) rats, 145 to $380 \mathrm{gm}$, were typically anesthetized with urethane $(1.4 \mathrm{gm} / \mathrm{kg}$, i.p.). The rats were not paralyzed or respirated. Whiskers were usually cut to a length of $20 \mathrm{~mm}$. The whisker movements indicated in the paper refer to the distance that the tip was moved. A 4-mm-diameter craniotomy was done over the somatosensory or visual cortex. The cortex was stained for 30 to $150 \mathrm{~min}$ by placing a pool of dye dissolved in saline $(0.9 \% \mathrm{NaCl})$ on the dura. The dye solution $(0.2$ to $4.0 \mathrm{mg} / \mathrm{ml})$ was then replaced with saline. Removing the dura (which is transparent in the rat) either before or after staining did not seem to affect signal size in a consistent way; most experiments were done with the dura intact. In two expcriments wo mcasured signal size (the change in fluorescence, $\Delta F$ ) as a function of time after the rinse. The signals were relatively small immediately after the rinse. After a 1 hr wait, the signals were larger. There was no change after another I-hr wait. One possible explanation for the slow increase is that, immediately after staining, a high concentration of dye is bound to the dura which would reduce the incident intensity reaching the cortex.
The rat was mounted on the microscope stage using a head-holder that had a clamp for the snout and ear bars. The use of ear bars reduced low frequency noise. The head holder allowed rotation about the rostral-caudal axis which was used to permit positioning of the surface of the cortex parallel to the microscope object plane. At the beginning of each whisker experiment, we focused on a region of corlex using the branching of the middle cerebral artery as a visual landmark and searched for signals using a large whisker deflection. If signals were found, the animal was moved in order to position the response area onto the desired portion of the array. Whiskers were moved by the arms of disassembled galvanometers driven by $30-\mathrm{msec}$ pulses from stimulators. The delay between the start of the stimulus current and the start of the whisker movement was measured. The arrows in the figures indicate the start of the whisker movement.

\section{Results}

Dye screening. Most dyes which had yielded large signals in other preparations were found to penetrate less than $50 \mu \mathrm{m}$ into the cortex when the cortex was sliced with a scalpel at the end of the staining period. A number of additional dyes were then tested for penetration, and those that penetrated more than $100 \mu \mathrm{m}$ were screened for signals in response to physiological stimuli. With most dyes the staining was darkest at the top of cortex and declined monotonically with depth. Staining with 10 dyes resulted in signals. The first group of dyes are analogues of the styryl, $\mathrm{RH} 414$ (Grinvald et al., 1984), the dye used most often in these experiments, which is in turn an analogue of dye 208 (Cohen et al., 1974) and the ASPPS dyes (Loew and Simpson, 1981). The structure of RH 414 is shown in Figure 3 . The styryls which gave signals were (concentration range in milligrams per milliliter in parentheses): $\mathrm{RH} 295(0.2)$, $\mathrm{RH} 414$ (0.3 to 6.0), $\mathrm{RH} 415$ (0.6), $\mathrm{RH} 425$ (0.6), $\mathrm{RH} 429$ (0.3 to 2.0), $\mathrm{RH} 437(0.3$ to 2.0$), \mathrm{RH} 461(0.3)$, and $\mathrm{RH} 493(0.1)$. Only a few oxonols, analogues of dye XXV (Gupta et al., 1981), were tested. RGA 422 (0.5 to 5.0) and RGA 448 (5.0) resulted in signals. Signals were found in 22 of the last 30 experiments using $\mathrm{RH} \mathrm{414;} \mathrm{because}$ of the variability in the size of the signals and the small number of experiments carried out with most dyes, additional testing of dyes and staining procedures may be useful.

Cortical responses to whisker movement. Figure 2 illustrates the results of an experiment in which 124 adjacent regions of cortex were simultaneously monitored. The traces are arranged so that their 


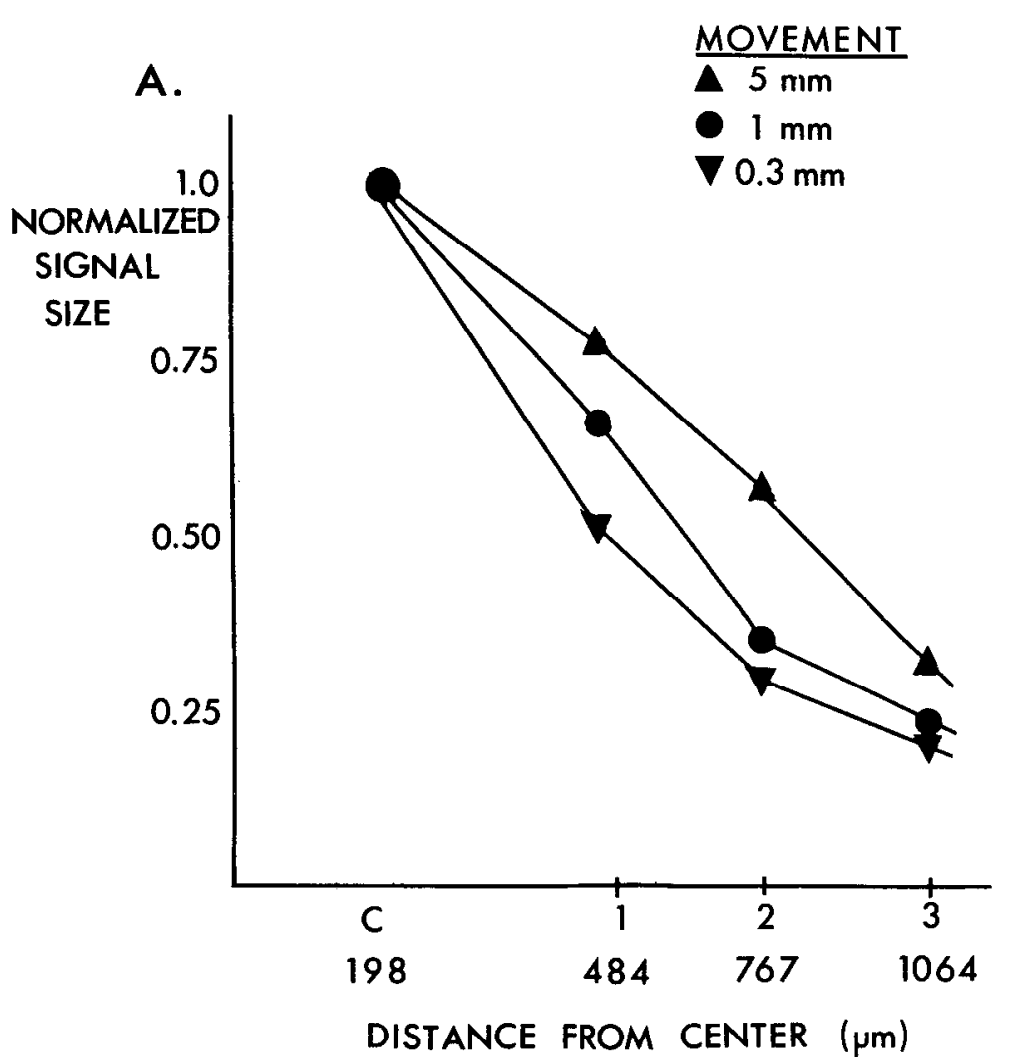

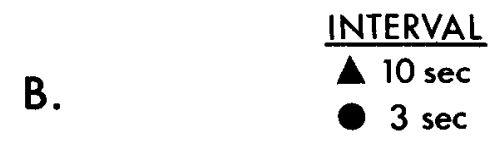

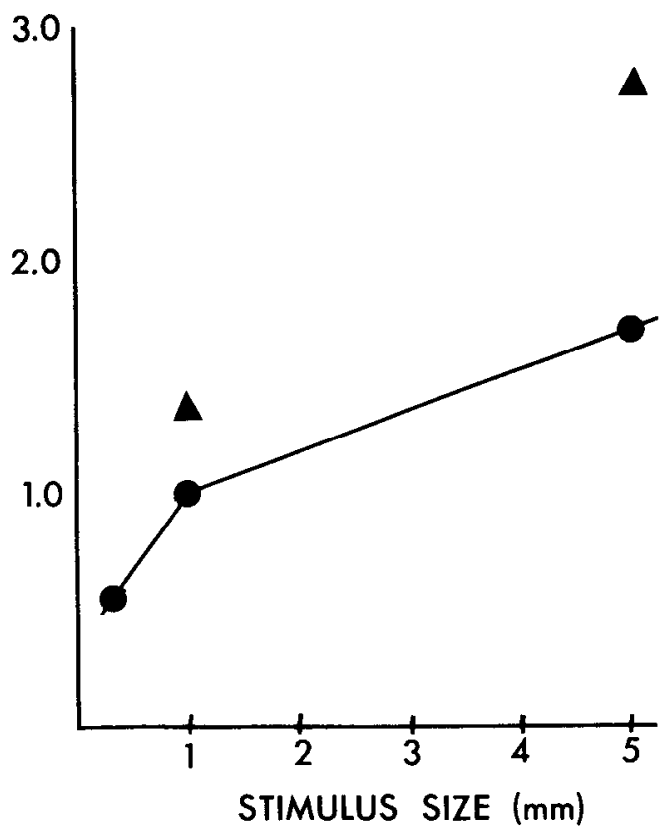

Figure 5. A, Signal size as a function of distance from the center of the response area. When the three curves were normalized at the central response, the fall-off was most rapid for the small stimulus and least rapid for the large stimulus. In five experiments we compared the shape of the response area using short $(2$ to $3 \mathrm{sec})$ and long $(6$ to $13 \mathrm{sec})$ interstimulus intervals. The interstimulus interval did not seem to affect the dimensions or shape of the response area. $B$, Signal size as a function of stimulus size and interstimulus interval. Larger stimuli led to larger signals in the center of the response area. (The 5-mm stimulus to one whisker caused a noticeable movement of an adjacent whisker.) Longer intertrial intervals also resulted in larger signals. Several preparations were used. For each preparation the result for movement of $1 \mathrm{~mm}$ and an interval of about $3 \mathrm{sec}$ (range, 2 to $3 \mathrm{sec}$ ) was normalized to 1.0 . The movement of $0.3 \mathrm{~mm}$ was compared to $1.0 \mathrm{~mm}$ in only one experiment. A $1-\mathrm{mm}$ movement was compared to a $5-\mathrm{mm}$ (range, 3 to $7 \mathrm{~mm}$ ) movement in seven preparations. The mean ratio of signal sizes from the large movement to the 1-mm movement was $1.65 \pm 0.11$ (SEM). The 3-sec intervals were compared to $10-\mathrm{sec}(6$ to $21 \mathrm{sec})$ intervals in three preparations. The ratios were $1.40 \pm 0.10$ for a 1 -mm movement and $1.68 \pm 0.05$ for a 5 -mm movement.

relative positions in the figure correspond to relative positions of the areas of the preparation imaged onto the detectors. The amount of light reaching a central element of the array was about $2 \times 10^{-2}$ $\mu \mathrm{W}$, or $5 \times 10^{7}$ photons/msec. When the tip of whisker B1 (for nomenclature, see Simons, 1978) was moved by $1 \mathrm{~mm}$, the number of photons reaching the central photodectors changed by 0.001 of the resting value $\left(\Delta F / F=10^{-3}\right)$. The time of the beginning of the whisker movement is given by the arrows under the traces in row 6 of Figure 2. A $4 \times$ objective was used; the centers of neighboring detectors received light from spots on the cortex that are separated by $283 \mu \mathrm{m}$. In all of the figures, a decrease in intensity is plotted upward. This direction of plotting was chosen because, in other preparations, depolarizations led to a decrease in intensity with styryl dyes of this type at this wavelength (Gupta et al., 1981; Grinvald et al., 1982b, 1984; Orbach and Cohen, 1983; Loew et al., 1985). In a few experiments we were able to detect signals in single trials, but most required averaging of several trials; 32 trials were averaged for Figure 2.

In the experiment illustrated in Figure 2, the delay between the start of the whisker movement and the start of the intensity change was measured without digital filtering on the averaged signal of the 16 central detectors and was $7 \mathrm{msec}$. The intensity change reached a peak about $50 \mathrm{msec}$ after the start of the movement and then returned toward the base line. To facilitate comparison of the time course of the signals, we combined the outputs of the central four detectors and combined each of the four concentric rings of detectors surrounding the central four. The arrangement of the central detectors (labeled $C$ ) and the four rings are shown in the inset of
Figure 3. In most of the 22 measurements that were analyzed, the signal at the center either had a shorter delay or had a more rapid rise than the signals from the edge of the response curve. Figure 3 illustrates the results of one such experiment in which the time courses of the central four, the second ring, and the fourth ring were scaled to the same peak height. The response from the fourth ring is delayed by about $8 \mathrm{msec}$ compared to the response from the central four. The response from the second ring has an intermediate time course. Thus, although the shapes of the signals throughout the response area were not dramatically different, the latency or rise time at the edge was often substantially slower.

In Figure $1 \mathrm{C}$, the signal that was filtered at 1.5 to $20 \mathrm{~Hz}$ had returned to the base line by the time of the next heartbeat, about $150 \mathrm{msec}$ after the start of the stimulus. This was our average result. However, since much of this decline toward the base line is caused by the low frequency cutoff of our amplifiers (compare DC-20 Hz with 1.5 to $20 \mathrm{~Hz}$ ), on average, the actual signal had not returned by 150 msec. The signals illustrated in Figures 2 and 4 are unusual in that they cross below the original base line by that time.

Controls. The signals presented here do not result from certain simple artifacts and are not due to light scattering. No signals were obtained if the staining or the stimulus was omitted, if the light was turned off, or if an excitation wavelength outside the absorption band of the dye was used. These results argue against a light scattering contribution and against coupling artifacts between the stimulus and the measuring system. However, the presence of fluorescent molecules in the cortex will increase the light intensity reaching the photodiodes, and one could suppose that this would 


\section{RAT VISUAL CORTEX}

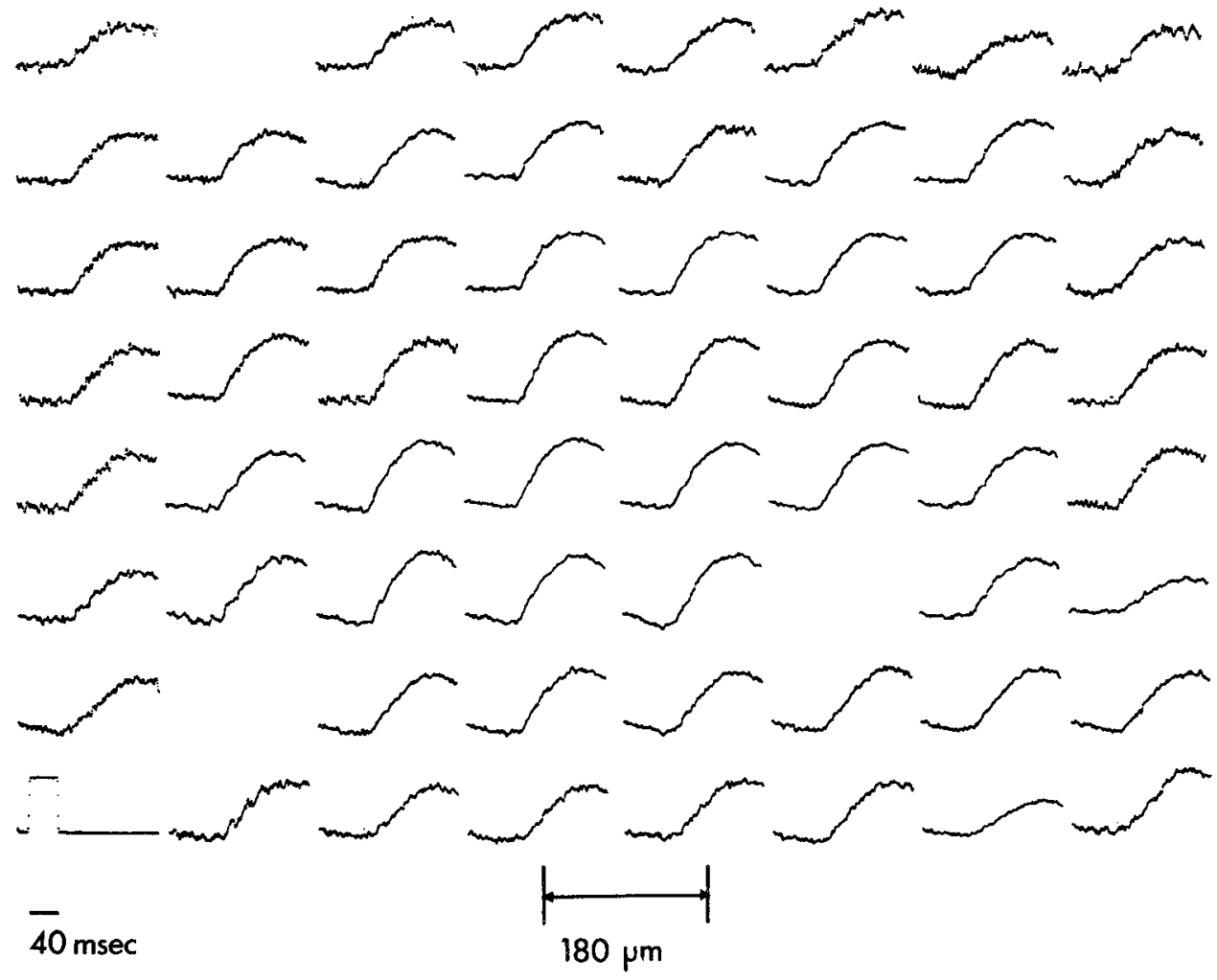

Figure 6. Responses to a flash of light to the contralateral eye measured in visual cortex. These signals are approximately uniform in size on all detectors, and occur with a longer delay and reach a peak more slowly than do the responses to whisker movement measured in somatosensory cortex. The outputs of the central 64 detectors of the $10 \times 10$ diode array are illustrated. Since these signals are relatively slow, their time course is more distorted by the low frequency filtering. The light flash to the eye was obtained by illuminating an electrome chanical shutter with a tungsten-filament bulb. A light guide was used to carry the light from the side of the shutter opposite the light source to within $1 \mathrm{~mm}$ of the eye. The eye had been bathed in mineral oil. The outputs of the individual detectors have been divided by the resting light level for this figure. The visual cortex was stained for $60 \mathrm{~min}$ with a $0.3-\mathrm{mg} / \mathrm{ml}$ solution of $\mathrm{RH} 414$. A total of 32 trials were averaged.

\section{INTERICTAL SPIKE}
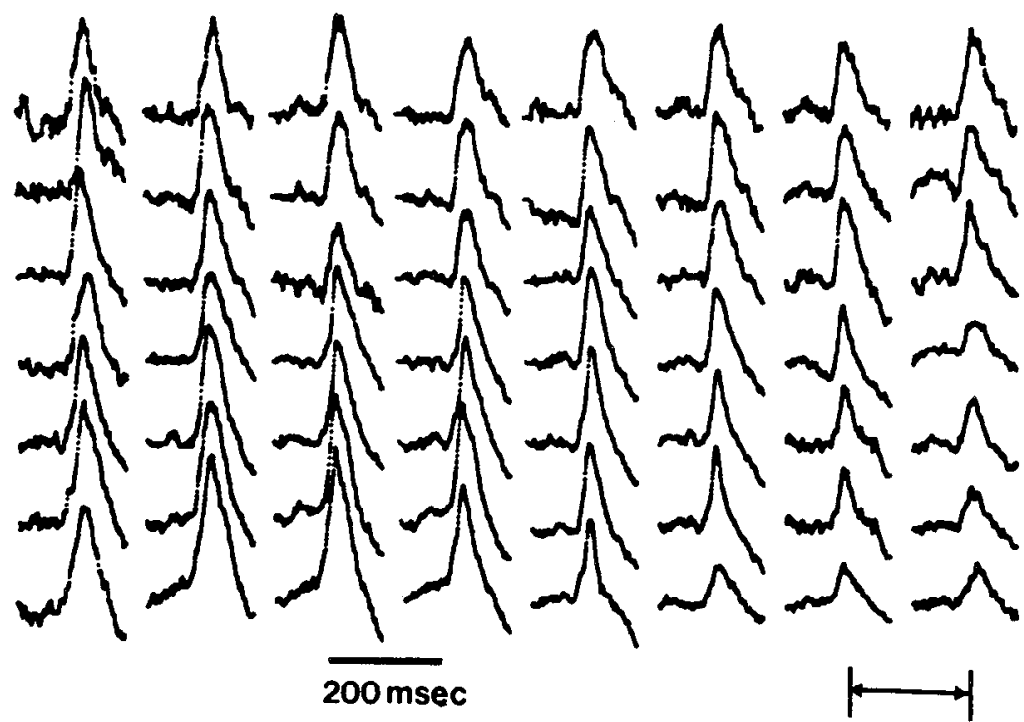

Figure 7. Spontaneous event induced by bicuculline. A large signal was found on all of the detectors in a single trial recording. The outputs of 56 of the photodiodes from the 10 $\times 10$ array are illustrated. The signals on the individual detectors have been normalized by the resting light level for this figure. The length of the vertical bar to the right of the figure represents a change in fluorescence divided by the resting fluorescence, $\Delta F / F$, of $5 \times 10^{-3}$. The visual cortex was stained for 60 min with a $1-\mathrm{mg} / \mathrm{ml}$ solution of $\mathrm{RH} 414$. The bicuculline solution (1 mM) was prepared as described by Zemon et al. (1980). The effect of bicuculline was transient; it was noticed within a minute or two after adding bicuculline and lasted for 5 to 15 min. Bicuculline was not used in the experiments illustrated in Figures 1 to 6.

enhance the possibility of detecting a light scattering signal. An additional result eliminates this possibility. If the signal was from scattering, it should be in the same direction no matter what dye was used. However, we found that styryl dyes gave signals that were decreases in intensity, whereas oxonols gave signals that were increases. This result is expected from previous experience with these dyes on other preparations (Gupta et al., 1981; Grinvald et al., 1981b, 1982b, 1984; Orbach and Cohen, 1983; Loew et al., 1985) but is inconsistent with a scattering origin of the signals.

We measured the signal at two incident wavelengths (filter 1). The fractional change at $540 \pm 15 \mathrm{~nm}$ was 2.2 times larger than the fractional changc at $480 \pm 15 \mathrm{~nm}$, a result that is consistent with measurements on squid axons (Loew et al., 1985; L. B. Cohen, A. Grinvald, and L. M. Loew, unpublished results). Thus, the signals have spectral characteristics in rat cortex that are similar to their behavior in other preparations-preparations in which it was demonstrated that the signals are dependent on changes in membrane potential. (This evidence, however, is not strong. For example, Beeler et al. (1981) found that relatively large changes in divalent ion concentration led to changes in the fluorescence of an oxonol dye 
(dye XXV of Gupta et al., 1981) in sarcoplasmic reticulum suspensions. This raises the possibility that, for example, $\mathrm{K}^{+}$accumulation in the extracellular space might lead to slow signals by affecting dye binding.)

Bleaching, photodynamic damage, and pharmacologic effects of the dyes. Repeated optical measurements led to a reduction in signal size. It took 200 to 400 trials, with the light on for $2 \mathrm{sec}$ in each trial, to reduce the signal to about half, although the time course of the signal was not dramatically affected. The reduction in size may be due to bleaching of the dye or to photodynamic damage resulting from the interaction of light, dye, oxygen, and neurons (Ross et al., 1977; Gupta et al., 1981), but it could also result from other causes, such as a time-dependent physiological deterioration of the preparation. In any case, since a repeated set of 32 trials usually led to signals of similar size and time course, photodynamic damage and bleaching were not large at the intensities we used.

The dyes used in these experiments have pharmacologic effects on other preparations when used at high concentration (Ross et al., 1977; Gupta et al., 1981; Grinvald et al., 1981a, 1982a, b, 1984). One control for pharmacologic effects was surface electrode recordings made before and after staining. In some experiments the evoked field potentials seemed to be unaffected by staining. In others, the evoked potential was smaller after staining, whereas in a few cases the evoked potential was larger after staining. In a few preliminary experinents measuring single urits in cal visual corlex with extracellular electrodes, staining did not seem to result in altered response characteristics of individual neurons (C. Gilbert and T. Wiesel, personal communication). Even though our information about pharmacologic effects is quite limited, it seems likely that the dyes are not strongly disrupting cortical function. Visual inspection of slices of cortex at the end of the experiments indicated that the amount of staining was often not large compared to some earlier invertebrate and tissue culture experiments (Salzberg et al., 1977; Grinvald et al., 1981b, 1982b) in which the staining of individual neurons was obvious. This result suggests that the concentration of dye per unitmembrane area may be relatively low in the present experiments.

Mapping responses to stimulation of two whiskers. Since the topography of the whiskers on the face corresponds to the topography of the whisker representation in cortex (Woolsey and Van Der Loos, 1970), the result in Figure 2 suggests that, if two whiskers, which are relatively far apart on the face, are stimulated, then signals would be found in separated areas of cortex. Figure 4 shows the results of such an experiment in which whisker D4 was stimulated early in the sweep and whisker A1 was stimulated late in the sweep (two arrows indicating the beginning of the two whisker movements are under each trace in row 6 of Fig. 4). There is a response on the right side of Figure 4 early in the sweep and a response on the left side late in the sweep. Such signals are not observed in the region between the two response areas. The centers of the two response areas are separated by about $2500 \mu \mathrm{m}$, a result that is in agreement with the separations found by Welker (1976) from electrode measurements on rat cortex.

Stimulus direction. In three preparations we compared the signals from caudal-rostral whisker movements with those obtained from rostral-caudal movements. In two preparations, rostral-caudal movements resulted in signals that were $50 \%$ larger; in the third, the signal size from the two movements was similar. In all three preparations the position and diameter of the response region on the cortex were unchanged when stimulus direction was changed.

Diameter and shape of the response area. We measured the diameter of the response areas by obtaining the peak size of the average signal for the central four detectors and the four surrounding rings. The results were plotted as a function of distance trom the center of the response area, and the width of the area at its halfheight was determined. For the experiment illustrated in Figure 2 the width was $1600 \mu \mathrm{m}$. This was somewhat larger than the mean of $1300 \mu \mathrm{m}$ for measurements on seven preparations for which whisker movements of 0.3 to $1.0 \mathrm{~mm}$ were used. The diameter of the signal area was affected by the size of the whisker movement. Figure $5 \mathrm{~A}$ illustrates the results from two preparations in which three movements were used. The signals were normalized to a size of 1.0 for the central four detectors. The diameter of the signal area was smallest for the small movement $(0.3 \mathrm{~mm})$ and largest for the large movement $(5 \mathrm{~mm})$.

Size of the signal on the central detectors. The size of the signal in the center of the response area was sensitive to two stimulus parameters. Figure $5 B$ is a plot of signal size versus whisker movement. Two interstimulus intervals are shown. Larger movements resulted in larger signals, and longer interstimulus intervals resulted in larger signals.

Inhibition. The finding that short interstimulus intervals resulted in smaller signals could be explained by some kind of inhibition (or adaptation or habituation) of a response that followed a prior stimulus. In related experiments we tried stimulating two different whiskers at different times in the trial, experiments similar to that illustrated in Figure 4. Seven repetitions of a three-measurement paradigm were carried out in three preparations. In each, a stimulus was given to whisker DI, and $60 \mathrm{msec}$ later a stimulus was given to the near neighbor, D3. Then, two control measurements were made, a stimulus to D1 alone and a stimulus to D3 alone. The computer was used to subtract the D1-alone result from the D1-D3 pair to give the D3 response when it is preceded by a D1 stimulus. This result was then compared with the measurement when D3 was stirriulaled alone. The difference is the amount by which the D3 response was altered as a result of the preceding D1 stimulus. In five of the seven experiments we detected inhibition that averaged $35 \%$. In two cases any inhibition was less than the noise in the measurement. The smallest inhibition we could detect was $25 \%$. This data tentatively suggest that stimulation of one whisker may inhibit the response to subsequent stimulation of a second whisker.

Light flash-Visual cortex. Diffuse flashes of light to the contralateral eye resulted in detectable signals from visual cortex. Figure 6 illustrates the response to a $40-\mathrm{msec}$ flash as measured on 80 detectors. The flash resulted in a signal that was approximately uniform in size over the entire cortical area $(1800 \times 1800 \mu \mathrm{m})$ imaged onto the photodiode array. We did not expect to see evidence of ocular dominance columns in rat cortex since this kind of organization was not detected in electrode measurements from mouse (Drager, 1978) or rat (U. Drager, unpublished observation). The responses in Figure 6 began with a much longer delay (55 msec) and reached a peak more slowly (200 msec) than did responses to whisker movement detected in somatosensory cortex. Furthermore, since the low frequency filtering will both reduce the size of the response and make the peaks appear artificially early, the actual peak must occur at a longer time after the stimulus.

Signals from epileptic events. We tested the effect of adding bicucultine, an agent thought to block cortical inhibition (Curtis et al., 1970). In some experiments, the bicuculline seemed to induce spontaneous events that have been termed "focal interictal epileptiform discharges" (Campbell and Holmes, 1983). Figure 7 illustrates the signals from 56 detectors in a measurement of one such spontaneous event in visual cortex. Since this is a single trial, and not an averaged result, it is clear that the interictal discharge leads to a much larger signal than does that obtained with whisker or visual stimulation in the absence of bicuculline. The fractional change, $\Delta r / r$, was $5 \times 10^{-3}$. This spontaneous signal was seen on all of the detectors of the array. The addition of bicuculline also increased the size of the responses evoked by whisker or visual stimulation, resulting in signals that were similar in size and time course to those shown in Figure 7.

Unusual signals. Signals of the sort illustrated in Figures 1 to 7 were found in experiments on 60 preparations. In three other preparations different results were obtained. In one experiment with $\mathrm{RH}$ 428 the signal was much briefer than usual (width at half-height of about $15 \mathrm{msec}$ ). In two experiments, using dyes $\mathrm{RH} 437$ and $\mathrm{RH}$ 295 , signals of reversed sign were found. A second experiment with 
$\mathrm{RH} 437$ resulted in signals in the usual direction. We did not investigate the origins of these unusual signals, but they could have resulted from intracellular accumulation of the dye which would then give a signal of opposite sign (Gupta et al., 1981).

\section{Discussion}

Origin of the signals. Two factors tend to emphasize signals from the upper layers of cortex. First, cortical light scattering will reduce the excitation intensity reaching deeper layers and will reduce the fluorescence from deeper layers that reaches the objective lens. If we assume that the cortex has the same scattering as that found in salamander olfactory bulb (no scattering measurements were made on cortex), then the signal-to-noise ratio for a constant amount of nerve activity would decrease by a factor of about 10 for each millimeter of thickness of cortex (Orbach and Cohen, 1983). Second, because the dye was introduced from the top of the cortex, the staining is darker in the upper layers. Because of these two factors, it seems most likely that the signals reported here originate in cortical layers I to III.

When illumination of the type illustrated in Figure 1 is used, knowing the depth at which the microscope objective is focused may, for certain signal source geometries, not be useful in determining the cortical layer responsible for the signal. If the neuron activity in a layer is uniform across the area of cortex imaged onto the array, then the optical signal at the diode array that results from this activity will be nearly independent of the depth at which the objective is focused. In the incident light path, a layer that is oul of focus will receive a reduced light intensity, but the lower light level will be compensated for by an increase in the area of the layer that is illuminated. A corollary argument holds for the intensity of the emitted light that reaches the diode array. This difficulty in determining the depth of the layer giving rise to the signal could be alleviated by using an illumination light path that is different from the measuring path which could be achieved by either using a Leitz vertical illuminator or by laser illumination at an oblique angle to the surface of the cortex. Another strategy would be to restrict the dye staining to a particular cortical layer. If, for instance, only the upper $150 \mu \mathrm{m}$ of cortex were stained either by brief staining periods or by using dyes which penetrate slowly, then not only would the layer responsible for the signal be known, but effects of scattering and out-offocus signals (see below) would be reduced. In this situation the spatial origins of the signals would be known with a resolution that was mainly determined by the magnification and photodetector size. If the optical signal arises from discrete areas within a layer, then its origin can be determined by determining the depth at which the largest, most compact signal is obtained (Salzberg et al., 1977).

Clearly the optical measurements we carried out are not providing single-unit resolution. The optical signals were not very different when a whisker was moved in opposite directions, whereas singleunit recordings showed that activity in some cortical neurons is strongly dependent on direction (Axelrad et al., 1976; Simons, 1978). The signals in somatosensory cortex started with a delay of 5 to 10 msec following the stimulus, whereas the signals from visual cortex had delays of 50 to $60 \mathrm{msec}$. These delays are consistent with the delays measured by Woolsey (1967) in evoked potential studies and may reflect differences in transducer and transmission times. Thus, rat signals began with longer delays, had simpler time courses, and had only a restricted spatial heterogeneity in time course when compared to the signals which were obtained from the rat hippocampal slices (Grinvald et al., 1982a), the salamander olfactory bulb (Orbach and Cohen, 1983), or the frog optic tectum (Grinvald et al., 1984). But the salamander olfactory bulb responses to odor stimulation (Kauer et al., 1984) had even longer latencies and also had uncomplicated time courses.

The explanation for the relative absence of temporal or spatial detail in the cortical and olfactory bulb signals is likely to be complex. Five factors are known which will tend to diminish detail in the optical signals. First, the individual detectors received light from relatively large areas of cortex, either $168 \times 168$ or $264 \times 264 \mu \mathrm{m}$ depending on the magnification used; thus, the light from many neurons will fall on each detector. The output of each detector will be some kind of average of the potential changes of all of the membranes (neuronal and glial) imaged onto the detector. A second factor that will smear signals results from the spread of both action potential and synaptic potential changes through dendritic and/or axonal arbors of neurons. Whereas single-unit recordings usually detect cell body spike activity and not dendritic signals, optical measurements would not make this distinction, and a signal would be detected over the entire cell. Two additional factors which will contribute to smearing the signals from discrele cortical areas are light scattering and signals from cortical layers that are out of focus. Measurements on the in vitro olfactory bulb (Orbach and Cohen, 1983) indicated that these factors smeared the light from a small spot over an apparent area of about $300 \mu \mathrm{m}$ in diameter; we will assume that the same amount of smearing holds for rat cortex. This area is about the same as the area of cortex imaged onto each detector when the $4 \times$ objective was used. With this magnification the optical smearing will cause light that should be on one detector to reach its near neighbors but not further.

Finally, we must consider the possibility that the bulb and cortical signals are smooth and slow because they, and not the slice and frog signals, result from the slow and long-lasting potential changes that are found in glial cells (Kelly and Van Essen, 1974). In a number of other preparations, voltage-sensitive dye signals have a time coursc very similar to that of simultaneous electrode recordings from neurons and have no slow signal (Ross et al., 1977; Salzberg et al., 1977, 1982; Gupta et al., 1981; Grinvald et al., 1981a; Ross and Krauthamer, 1984) that might be attributed to a potential change in a glial cell or Schwann cell that was dependent on the neuron's activity. In contrast, two instances of unexpected, slow optical signals have been reported (Grinvald et al., 1981a; Salzberg et al., 1983; Lev-Ram and Grinvald, 1984), and glial origins have been suggested. However, in salamander olfactory bulb, action potentials in olfactory axons and nerve terminals are not followed by slow signals (second stimulus, Fig. 5; Orbach and Cohen, 1983), thus, the only evidence from optical measurements in vertebrate CNS shows that neuron activity is not always followed by detectable signals from glia. In addition, the glia responses to visual stimuli found in visual cortex (Kelly and Van Essen, 1974) had rise times of about $1 \mathrm{~min}$, a time course much too slow to be detected by our AC-coupled measuring system.

Size of the response area. Using the scattering measurement from olfactory bulb to correct our results, we estimate, as a first approximation, that the actual width of the response area to a small whisker movement was $1000 \mu \mathrm{m}$ ( $300 \mu \mathrm{m}$ smaller than the measured mean width of $1300 \mu \mathrm{m}$ ). The estimate of $1000 \mu \mathrm{m}$ is at least 2 times larger than the diameter of the barrel measured anatomically at layer IV. However, a result in this direction is not unexpected since many neurons in layers $I$ and III respond to stimulation of more than one whisker (Axelrad et al., 1976; Simons, 1978). Simons (1978) found that more than $40 \%$ of the units he recorded in these layers were activated by more than one whisker. Furthermore, a neuron that responded to only one whisker might have processes in more than one barrel. Both factors will tend to make the optical response area larger than the anatomically defined barrel.

Our finding that smaller stimuli resulted in smaller response area (Fig. 5A) confirms the results of Axelrad et al. (1976), which were obtained from sequential surface field potential measurements. The interpretation of optical signals may be simpler than the interpretation of field potentials. The optical measurement is, as far as is known, determined by membrane potential. However, the signal recorded in a field potential measurement has a complex relationship to the membrane potential of the active cell population and to the position of the recording electrodes (Rall and Shepherd, 1968; Llinas and Nicholson, 1974). The extracellular potential may be proportional to either membrane potential or, more often, to its first or second 
derivative. Assuming uniform dye binding, the signal size of an optical measurement will be proportional to the membrane area of the cells imaged on the detector, whereas the field potential measurement will give a result that is more biased toward large processes and neurons. The optical signal will be nearly insensitive to neuronal geometry or orientation, whereas a field potential measurement is very sensitive to these factors. Finally, the spatial origins of our optical signals are known to within a few hundred micrometers, whereas the origin of a field potential signal can be located only with difficulty. Thus, a comparison of optical measurements with field potential measurements suggests that, at present, the two provide different but complementary information.

Using signal size during an interictal discharge (Fig. 7), we can make, with assumptions, a very rough estimate of the percentage of activated neurons necessary to generate the signals obtained in the experiment illustrated in Figure 2. First, we assume that the interictal discharge results from an average depolarization of $30 \mathrm{mV}$ of all of the neurons and a $10-\mathrm{mV}$ depolarization of all of the glial cells (Gutnick et al., 1982), that $70 \%$ of the membrane in cortex is neuronal and $30 \%$ is glial, and that all of the signal in Figure 2 originates from neurons. Then, we further assume that the linearity of optical signal with membrane potential found in other preparations (Cohen and Salzberg, 1978) holds for experiments in cortex, and that the signal size is proportional to the percentage of activated ncurons. Since the fractional fluorescence changc at the pcak of the interictal discharge (Fig. 7) was about 5 times that found in Figure 2, the peak signal in Figure 2 could be accounted for by a $20-\mathrm{mV}$ depolarization in $25 \%$ of the neurons or a very high firing rate of a smaller percentage of neurons. Thus, activation of a substantial fraction of the neurons may be necessary to give rise to signals of the size we obtained.

Preliminary experiments were conducted together with C. Gilbert and $T$. Wiesel in an attempt to measure signals from cat visual cortex in response to visual stimulation. Signals were found after the addition of bicuculline, but none were found in untreated cortices. There are several explanations that might account for smaller signals in the cat experiment. One is that our stimulus generated a smaller net activation of cat cortical neurons. Another is that dyes which were satisfactory on the rat might not work as well on the cat.

The response to whisker movement was graded in almost every characteristic that we measured. The size of the signal was a graded function of the size of the stimulus. The cortical area involved in the response was a graded function of the stimulus size. The time course of the signal was faster at the center of the response area than it was at the edges. Finally, the size of the response seemed to depend on the previous stimulation history of the whisker or its neighbors. Thus, although the whisker barrel may appear to be fixed in terms of its anatomy, the responses we measured changed with variations in stimulus parameters.

Our experiments demonstrate that optical measurements can simultaneously detect activity-related signals from 100 to 124 sites on cortex with a time resolution of milliseconds and a spatial resolution of a few hundred micrometers. Dramatic improvements in the signal-to-noise ratio and spatial resolution and a large increase in the number of simultaneously monitored areas may be possible. Preliminary experiments carried out on cat visual cortex suggest that much of the low frequency noise remaining after the heartbeat subtraction procedure can be removed by temporarily stopping the breathing of an artificially respired animal. Once the movement noise is eliminated, the effects of the remaining noise, dark noise and shot noise, can be reduced by increasing the incident light intensity. In addition, better dyes may be found or designed. Finally, modification of the optics should reduce the effects of scattering and the contribution from out-of-focus layers and thereby improve resolution.

\section{References}

Axelrad, H., R. Verley, and E. Farkas (1976) Responses evoked in mouse and rat Si cortex by vibrissa stimulation. Neurosci. Lett. 3: 265-274.
Beeler, T. J., R. H. Farmen, and A. N. Martinosi (1981) The mechanism of voltage-sensitive dye responses on sarcoplasmic reticulum. J. Membr. Biol. 62: 113-137

Campbell, A. M., and O. Holmes (1983) Bicuculline epileptogenesis in the rat. J. Physiol. (Lond.) 342: $41 \mathrm{P}$

Cohen, L. B., B. M. Salzberg, H. V. Davila, W. N. Ross, D. Landowne, A. S. Waggoner, and C. H. Wang (1974) Changes in axon fluorescence during activity: Molecular probes of membrane potential. J. Memb. Biol. 19: 1 36

Cohen, L. B., and B. M. Salzberg (1978) Optical measurement of membrane potential. Rev. Physiol. Biochem. Pharmacol. 83: 35-88.

Cohen, L. B., B. M. Salzberg, and A. Grinvald (1978) Optical methods for monitoring neuron activity. Annu. Rev. Neurosci. 1: 171-182.

Curtis, D. R., A. W. Duggan, D. Felix, and G. A. R. Johnston (1970) GABA, bicuculline and central inhibition. Nature 226: 1222-1224.

Drager, U. C. (1978) Observations on monocular deprivation in mice. J. Neurophysiol. 41: 28-42.

Grinvald, A. (1985) Real-time optical mapping of neuronal activity: From single growth cones to the intact mammalian brain. Annu. Rev. Neurosci. 8: 263-305.

Grinvald, A., L. B. Cohen, S. Lesher, and M. B. Boyle (1981a) Simultaneous optical monitoring of activity of many neurons in invertebrate ganglia using a 124 element photodiode array. J. Neurophysiol. 45: 829-840.

Grinvald, A., W. N. Ross, and I. C. Farber (1981b) Simultaneous optical measurements of electrical activity from multiple sites on processes of cultured neurons. Proc. Natl. Acad. Sci. U. S. A. 78: 3245-3249.

Grinvald, A., A. Manker, and M. Segal (1982a) Visualization of the spread of electrical activity in rat hippocampal slices by voltage-sensitive optical probes. J. Physiol. (Lond.) 333: 269-291.

Grinvald, A., R. Hildesheim, I. C. Farber, and L. Anglister (1982b) Improver fluorescent probes for the measurement of rapid changes in membrane potential. Biophys. J. 39: 301-308

Grinvald, A., L. Anglister, J. A. Freeman, R. Hildesheim, and A. Manker (1984) Real-time optical imaging of naturally evoked electrical activity in intact frog brain. Nature 308: 848-850.

Gupta, R. K., B. M. Salzberg, A. Grinvald, L. B. Cohen, K. Kamino, S. Lesher M. B. Boyle, A. S. Waggoner, and C. H. Wang (1981) Improvements in optical methods for measuring rapid changes in membrane potential. $\mathrm{J}$. Membr. Biol. 58: 123-137.

Gutnick, M. J., B. W. Connors, and D. A. Prince (1982) Mechanisms of neocortical epileptogenesis in vitro. J. Neurophysiol. 48: 1321-1335.

Hamming, R. W. (1977) Digital Filters, Prentice-Hall, Inc., Englewood Cliffs, NJ.

Kauer, J. S., D. Senseman, and L. B. Cohen (1984) Voltage-sensitive dye recording from the olfactory system of the tiger salamander. Soc. Neurosci. Abstr. 10: 846 .

Kelly, J. P., and D. C. Van Essen (1974) Cell structure and function in the visual cortex of the cat. J. Physiol. (Lond.) 238: 515-547.

Lev-Ram, V., and A. Grinvald (1984) is there a potassium dependent depolarization of the paranodal region of myelin sheath? Optical studies of rat optic nerve. Soc. Neurosci. Abstr. 10: 948.

Llinas, R., and C. Nicholson (1974) Analysis of field-potentials in the central nervous system. In Handbook of Electroencephalography and Clinical Neurophysiology, C. F. Stevens, ed., Vol. 2, Part B, pp. 61-92, Publisher, City.

Loew, L. M., and L. L. Simpson (1981) Charge shift probes of membrane potential, a probable electrochromic mechanism for p-aminostyryl pyridinium probes on a hemispherical lipid bilayer. Biophys. J. 34: 353-365.

Loew, L. M., L. B. Cohen, B. M. Salzberg, A. L. Obaid, and F. Bezanilla (1985) Charge shift probes of membrane potential. Characterization of amininostyrylpyridinium dyes on the squid giant axon. Biophys. J. 47: 7177

Orbach, H. S., and L. B. Cohen (1983) Optical monitoring of activity from many areas of the in vivo and in vitro salamander olfactory bulb: A new method for studying functional organization in the vertebrate central nervous system. J. Neurosci. 3: 2251-2262.

Orbach, H. S., L. B. Cohen, and A. Grinvald (1982) Optical monitoring of evoked activity in the visual cortex of the marine rat. Biol. Bull. 163: 389.

Orbach, H. S., L. B. Cohen, A. Grinvald, and R. Hildesheim (1983) Optical monitoring of neuron activity in rat somatosensory and visual cortex. Soc. Neurosci. Abstr. 9: 39.

Rall, W., and G. M. Shepherd (1968) Theoretical reconstruction of field potentials and dendrodendritic synaptic interactions in olfactory bulb. $\mathrm{J}$. Neurophysiol. 31: 884-915.

Ross, W. N., and V. Krauthamer (1984) Optical measurements of potential 
changes in axons and processes of neurons of a barnacle ganglion. $J$ Neurosci. 4: 659-672.

Ross, W. N., B. M. Salzberg, L. B. Cohen, A. Grinvald, H. V. Davila, A. S. Waggoner, and C. H. Wang (1977) Changes in absorption, fluorescence, dichroism, and birefringence in stained giant axons: Optical measurement of membrane potential. J. Membr. Biol. 33: 141-183.

Salzberg, B. M. (1983) Optical recording of electrical activity in neurons using molecular probes. In Current Methods in Cellular Neurobiology, J. L. Barker and J. F. McKelvy, eds., pp. 139-187, John Wiley \& Sons, Inc., New York.

Salzberg, B. M., A. Grinvald, L. B. Cohen, H. V. Davila, and W. N. Ross (1977) Optical recording of neuronal activity in an invertebrate central nervous system: Simultaneous monitoring of several neurons. d. Neurophysiol. 40: 1281-1291.

Salzberg, B. M., A. L. Obaid, H. Shimizu, R. K. Orkand, and D. M. Senseman (1982) Does the Schwann cell of Loligo act as a potassium electrode? Optical studies using potentiometric probe. Biol. Bull. 163: 390.

Salzberg, B. M., A. L. Obaid, D. H. Senseman, and H. Gainer (1983) Optical recording of action potentials from vertebrate nerve terminals using potentiometric probes provides evidence for sodium and calcium components. Nature 306: 36-40.

Simons, D. J. (1978) Response properties of vibrissa units in rat Sl somatosensory cortex. J. Neurophysiol. 41: 798-820.

Waggoner, A. S. (1979) Dye indicators of membrane potential. Annu. Rev. Biophys. Bioeng. 8: 47-68.

Welker, C. (1976) Receptive fields of barrels in the somatosensory neocortex of the rat. J. Comp. Neurol. 166: 173-190.

Woolsey, T. A. (1967) Somatosensory, auditory and visual cortical areas of the mouse. Johns Hopkins Med. J. 121: 91-112.

Woolsey, T. A., and H. Van Der Loos (1970) The structural organization of layer IV in the somatosensory region (SI) of mouse cerebral cortex. The description of a cortical field composed of discrete cytoarchitectonic units. Brain Res. 17: 205-242.

Zemon, V., E. Kaplan, and F. Ratliff (1980) Bicuculline enhances a negativo component and diminishes a positive component of the visual evoked cortical potential in the cat. Proc. Natl. Acad. Sci. U. S. A. 77: 7476-7478. 